\title{
Large Vessel Occlusion Stroke Secondary to Acute Aortic Dissection
}

\author{
Alec J. Pawlukiewicz ${ }^{1}$, Drew Long ${ }^{1}$, Sumeru Mehta ${ }^{2}$ \\ 1. Emergency Medicine, Brooke Army Medical Center, Fort Sam Houston, USA 2. Emergency Medicine, Greater San \\ Antonio Emergency Physicians, San Antonio, USA
}

Corresponding author: Alec J. Pawlukiewicz, ajpawlukiewicz@gmail.com

\begin{abstract}
Aortic dissection carries a high mortality of up to $40 \%$ at the time of initial dissection and an additional $1 \%$ per hour the dissection is untreated. Patients with acute aortic dissection most commonly present with chest or back pain. Less frequently, it manifests without pain with predominant neurologic symptoms secondary to an acute stroke. We present the case of a 53-year-old male presenting with acute onset aphasia and rightsided weakness. Incidentally, CT angiography of his neck revealed a carotid artery dissection, which was found an extension of a Stanford type A acute aortic dissection resulting in a large vessel occlusion stroke. The patient's concomitant pathologies resulted in uncertainty as to the priority of management between the interventional neurology and cardiothoracic surgery services, ultimately resulting in the transfer of the patient to an aorta specialist at an outside facility. This case highlights several areas of difficulty in the management of patients with presenting with both large vessel occlusion stroke and acute aortic dissection and the need for consideration of acute aortic dissection in patients presenting with symptoms consistent with large vessel occlusion stroke. Optimal blood pressure control is unknown, as is the ideal timing of aortic repair and the potential for endovascular therapy for large vessel occlusion stroke in the setting of acute aortic dissection. Emergency physicians must rapidly engage with neurology, interventional neurology, and cardiothoracic surgery to determine appropriate interventions and timing of operative repair. The emergency physician must consider acute aortic dissection in patients presenting with signs and symptoms concerning for large vessel occlusion stroke, even if they have no complaint of chest pain, as administration of thrombolytics in these patients may be deadly.
\end{abstract}

Received 06/26/2020

Review began 07/04/2020 Review ended 07/12/2020 Published 07/19/2020

๑) Copyright 2020

Pawlukiewicz et al. This is an open access article distributed under the terms of the Creative Commons Attribution License CC-BY 4.0., which permits unrestricted use, distribution, and reproduction in any medium, provided the original author and source are credited.
Categories: Cardiac/Thoracic/Vascular Surgery, Emergency Medicine, Neurology

Keywords: aortic dissection, stroke, large vessel occlusion, endovascular therapy, thrombolysis, chest pain

\section{Introduction}

Aortic dissection $(\mathrm{AD})$ is a disruption of the media layer of the aorta, typically secondary to an intimal tear, resulting in bleeding within the aortic wall and subsequent separation of the three layers of the aorta [1]. $\mathrm{AD}$ can be classified anatomically by the location of the intimal tear (DeBakey classification) or by whether the ascending aorta is involved (Stanford classification). AD is an uncommon but potentially deadly condition with an incidence of 2-3.5 per 100,000 person-years, with a mortality up to $40 \%$ at the time of initial dissection [1-4]. Furthermore, it carries an increasing mortality of $1 \%$ per hour the dissection is untreated, emphasizing the necessity for high suspicion and rapid detection by the emergency physician (EP). Unfortunately, $\mathrm{AD}$ is often difficult to diagnose on initial presentation. $\mathrm{AD}$ classically presents as ripping or tearing severe chest pain that is sudden in onset with radiation to the back [5,6]. Presenting less commonly as a focal neurologic deficit, acute $\mathrm{AD}$ may lead to an ischemic stroke through various mechanisms. We present the case of a 53-year-old male presenting to our emergency department (ED) with right-sided weakness and aphasia, found to have an extensive acute $\mathrm{AD}$ leading to a large vessel occlusion (LVO) ischemic stroke.

\section{Case Presentation}

A 53-year-old male with a history of atrial fibrillation and an aortic aneurysm presented to our ED by Emergency Medical Services (EMS) with acute onset of right-sided weakness. Per EMS report, he was found to have right-sided weakness, aphasia, and left gaze preference approximately one hour prior to arrival. Initial vital signs upon arrival included an irregular heart rate (HR) of 99 beats per minute (bpm), blood pressure (BP) of 155/78 $\mathrm{mmHg}$, a respiratory rate (RR) of 21 breaths per minute, a temperature of $97.4^{\circ} \mathrm{F}$, and an oxygen saturation of 100\%. A National Institutes of Health Stroke Scale (NIHSS) of 16 was calculated during initial evaluation with points given for inability to perform simultaneous tasks, aphasia, right upper extremity weakness, right lower extremity weakness, and gaze deviation. A stroke alert was called, and the neurology service was consulted. The patient's electrocardiogram (ECG) showed atrial fibrillation with a rate of 83 without other significant features (Figure 1). 


\section{Cureus}

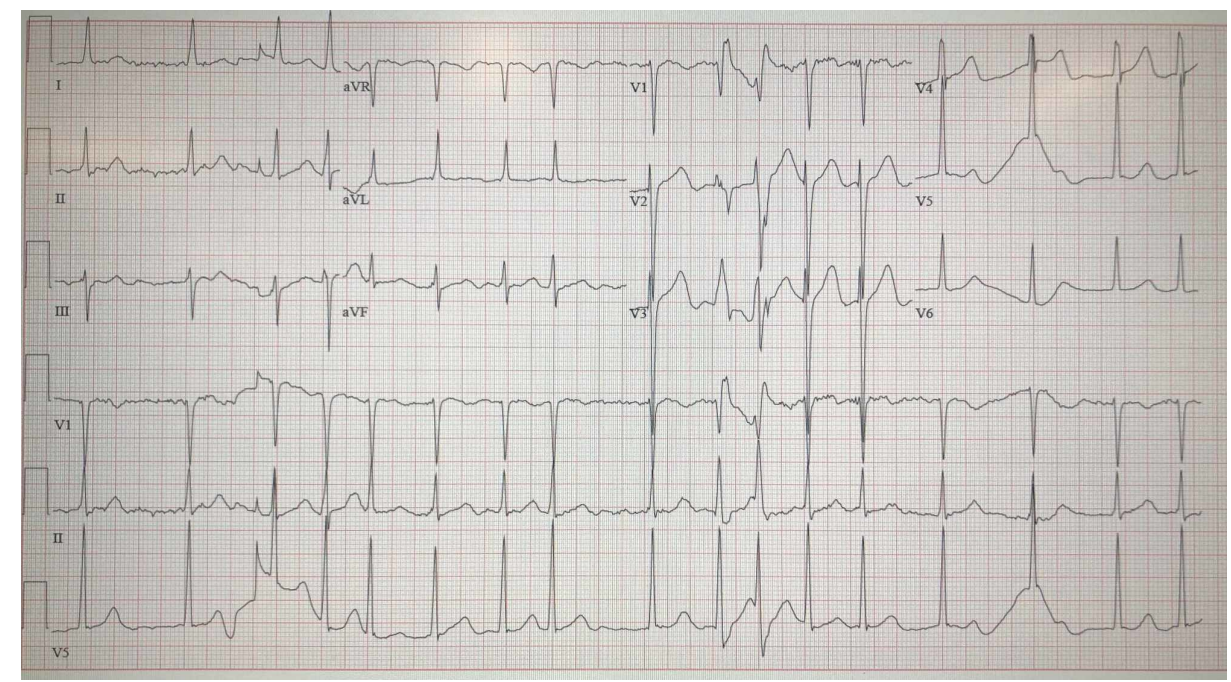

FIGURE 1: Electrocardiogram demonstrating atrial fibrillation with a rate of 83.

Chest x-ray showed a widened mediastinum with enlarged aortic contour (Figure 2). CT noncontrast of the head and CT angiography (CTA) of the head and neck showed findings consistent with an early middle cerebral artery (MCA) infarct with an embolus to the M1 segment of the left MCA with extension to the proximal M2 segments, in addition to dissection of the left internal carotid artery extending into the left subclavian artery (Figure 3).

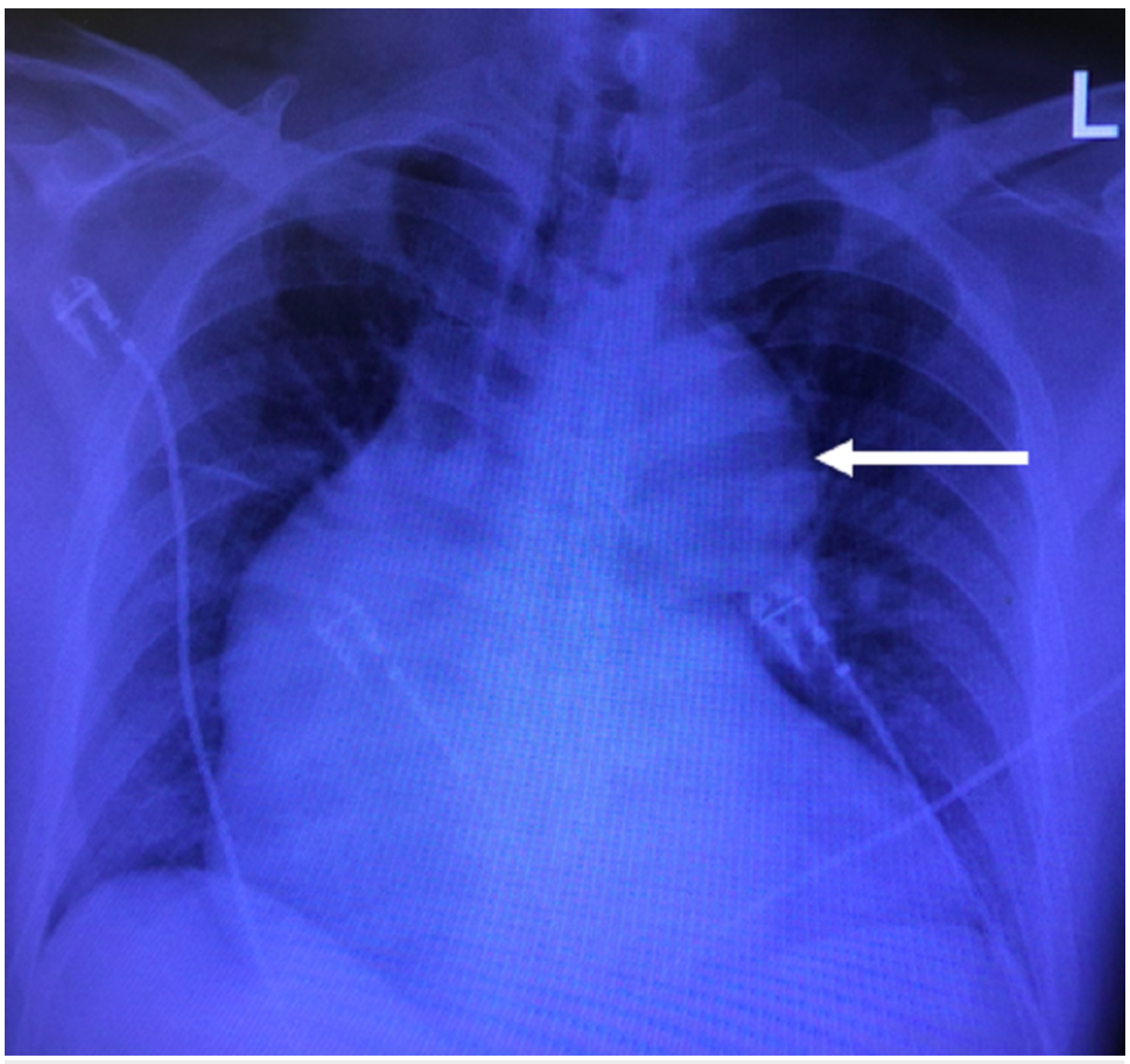

FIGURE 2: Chest x-ray with a widened mediastinum and abnormal aortic contour, indicated by the white arrow. 


\section{Cureus}

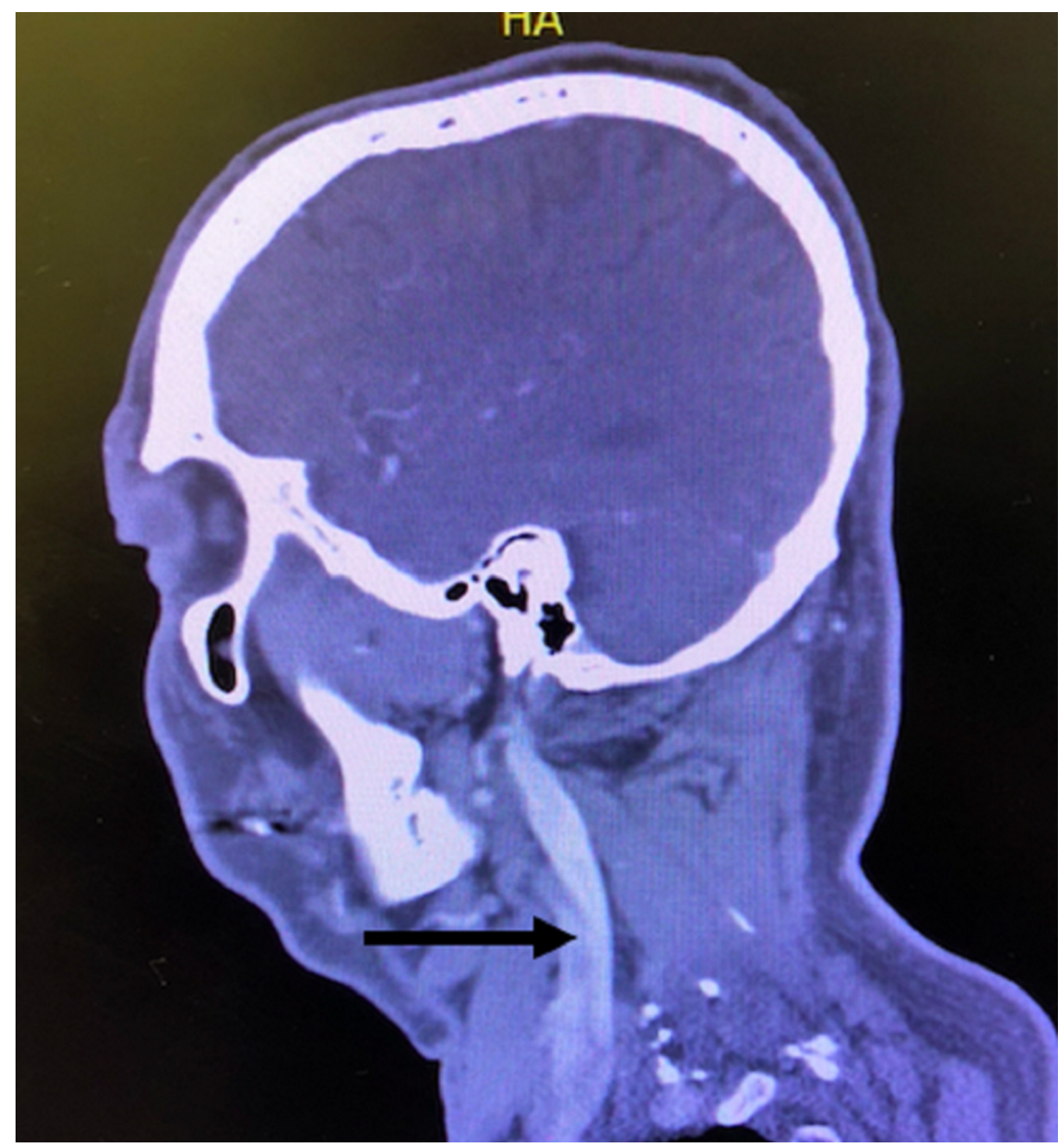

FIGURE 3: Sagittal view of CT angiography of the neck showing a left internal carotid artery dissection, with an arrow indicating the dissection.

Given concern for an AD based on the chest X-ray and left internal carotid artery dissection, CTA of the aorta was obtained. CTA showed an ascending thoracic aneurysm with Stanford type A dissection from the aortic valve to the level of the renal arteries with extension to the left internal carotid artery (Figures 4,5 ). 


\section{Cureus}

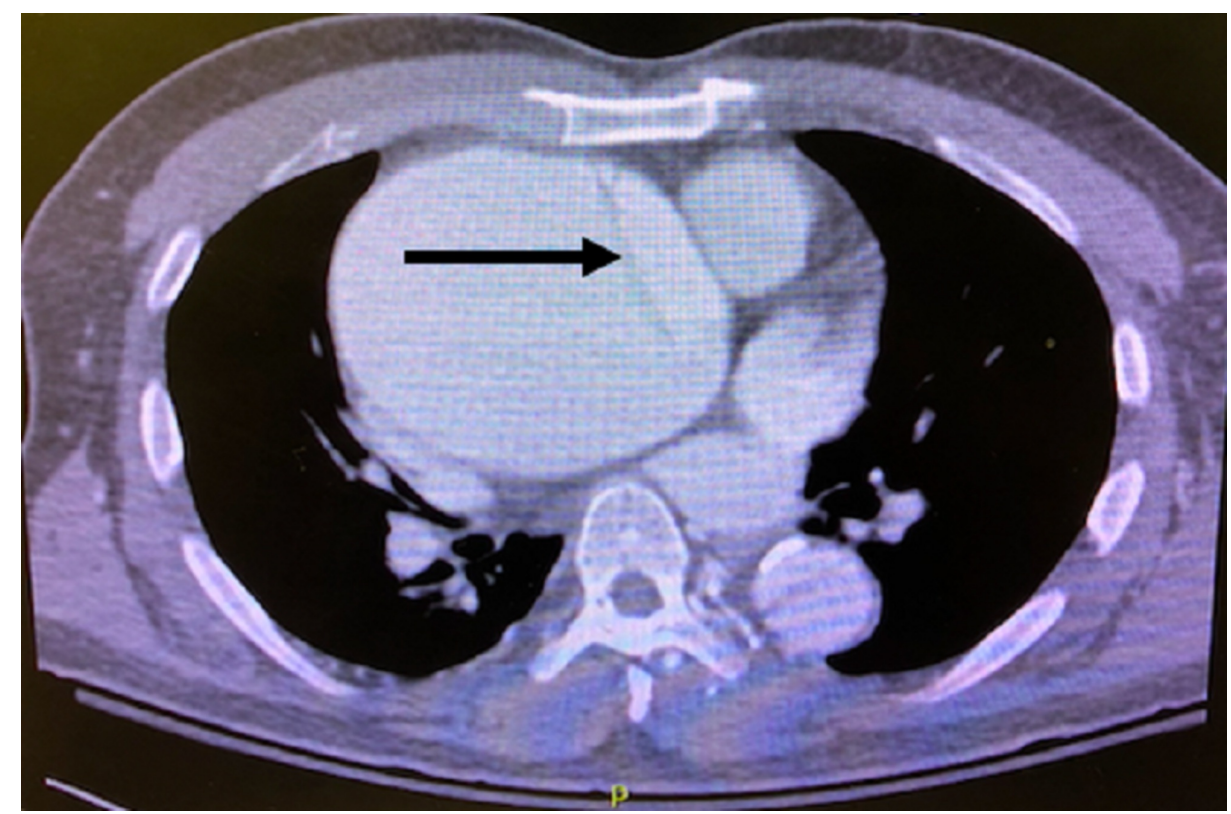

FIGURE 4: Transverse view of CT angiography of the chest showing a large aortic aneurysm with dissection, with an arrow delineating the dissection flap.

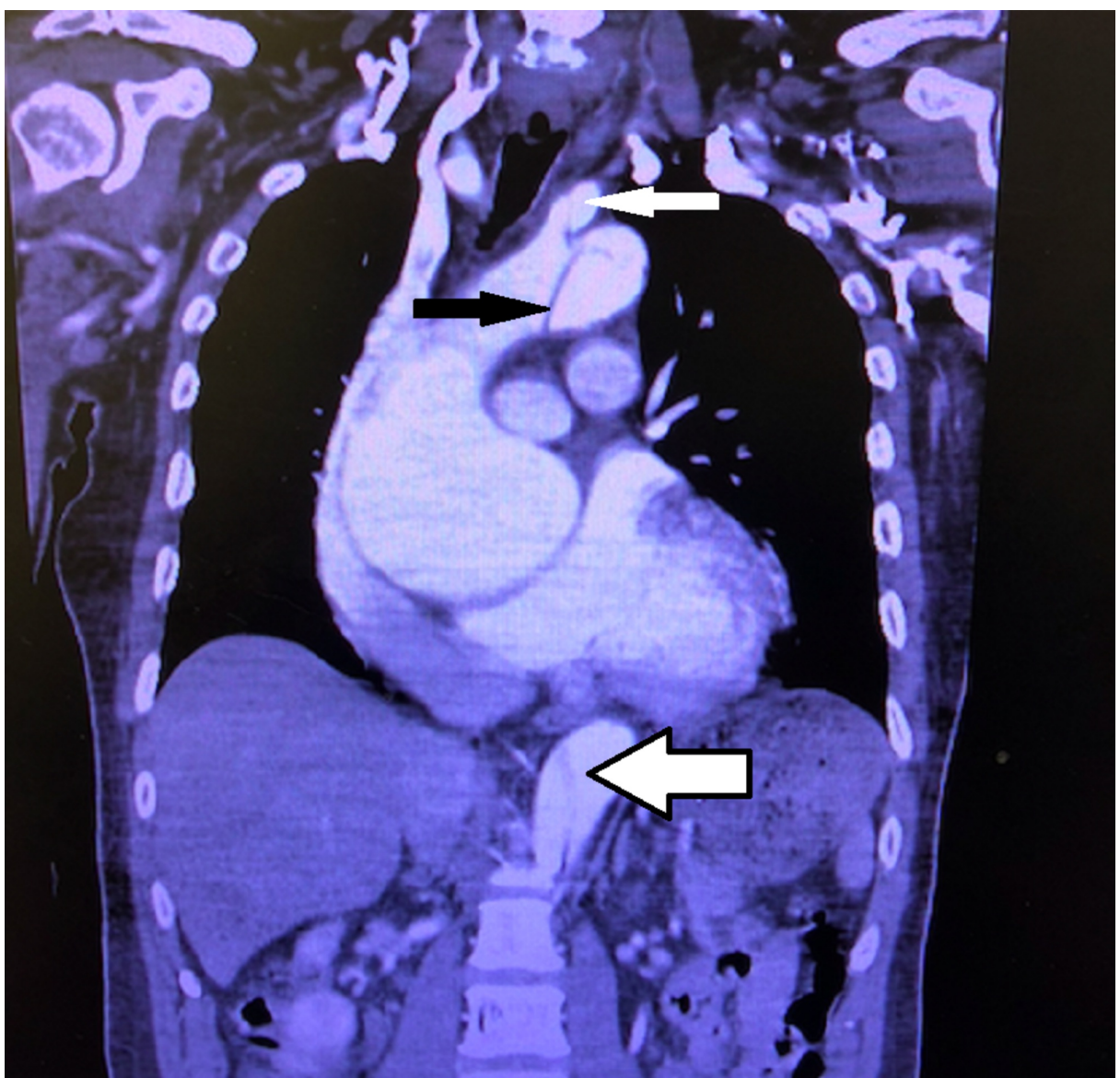

FIGURE 5: Coronal view of CT angiography of the chest showing a large aortic aneurysm with dissection, with a solid black arrow depicting the dissection in the ascending aorta, a solid white arrow depicting the dissection in the left internal carotid artery, and a white arrow with 
black outline showing the dissection of the descending aorta.

The patient was started on esmolol and nicardipine intravenous drips to optimize heart rate and $\mathrm{BP}$ with a goal heart rate of $60 \mathrm{bpm}$ and goal systolic BP of $120 \mathrm{mmHg}$. The case was discussed with the neurology and cardiothoracic surgery services. Given the finding of an LVO stroke, the interventional neurology service was also consulted. The consulting physicians did not think the patient was an optimal candidate for either immediate endovascular therapy or surgical aortic repair. In conjunction with cardiothoracic surgery, the decision was made to transfer the patient to a center with an aortic specialist for operative repair. The patient was maintained on the esmolol and nicardipine infusions and in stable condition until the time of transfer. While in the ED, the patient's neurologic exam was unchanged with persistence of his right hemiplegia, aphasia, and left gaze preference. He was ultimately transferred to a center for potential definitive care of his $\mathrm{AD}$ and was lost to follow-up.

\section{Discussion}

Given the high morbidity and mortality, the EP must rapidly identify the patient with AD. Unfortunately, AD is challenging to diagnose. The most common complaints in patients with $\mathrm{AD}$ are chest and/or back pain, usually sudden and severe in onset $[5,6]$. However, patients often present atypically, and one retrospective review found $6.4 \%$ of patients presented without chest or back pain [7]. Of patients whose $\mathrm{AD}$ is associated with neurologic symptoms (17\%-29\%), approximately $53 \%$ have ischemic stroke as their causative etiology $[8,9]$. Patients with neurologic symptoms secondary to $\mathrm{AD}$ may be especially difficult to diagnose, as up to one-third of patients presenting with neurologic features do not complain of chest pain $[1,9]$. Initial assessment may be further complicated by aphasia, amnesia, or altered mental status, clouding an accurate history, as seen in the described case [8,9]. While neurologic symptoms may confound the diagnosis, a metaanalysis from 2018 on the history and exam features predictive of acute $\mathrm{AD}$ found the clinical signs most suggestive for acute $\mathrm{AD}$ were focal neurologic deficits, pulse deficits, and hypotension ( $\mathrm{BP}<90 \mathrm{mmHg}$ ) or signs of shock [10]. The EP must consider AD in patients with focal neurologic symptoms concerning for an ischemic stroke, especially if they are aphasic or altered and unable to give a history.

Optimal management of patients presenting with ischemic stroke secondary to acute $\mathrm{AD}$ is unknown. The co-occurrence of ischemic stroke and $\mathrm{AD}$ increases the possibility of complications during management for several reasons. Current emergency medicine guidelines support the administration of thrombolytics to patients presenting with acute ischemic stroke within 3 to 4.5 hours of symptom onset in patients without contraindications [11]. While there is a paucity of literature concerning thrombolytics administration in patients with ischemic stroke and acute $\mathrm{AD}$, a review of the effect of thrombolytics given for myocardial infarction in patients found to have ADs showed an association with increased morbidity and mortality [12]. As giving thrombolytics to a patient with an acute $\mathrm{AD}$ may lead to worsening bleeding within the false lumen and progression of the dissection, acute $\mathrm{AD}$ is a contraindication to administration of thrombolytics.

A relatively newer therapy for management of ischemic stroke patients is endovascular therapy. Endovascular therapy has been shown to lead to improved outcomes and decreased complications in select patients with LVO strokes up to 24 hours from the onset of symptoms [13-15]. However, there are limited data on endovascular therapy for LVO stroke in the setting of AD. One case series from 2017 identified three patients with proximal right MCA occlusions in the setting of $\mathrm{AD}$, all of which underwent successful recanalization with endovascular therapy and experienced improved clinical neurologic status [16]. However, two of these patients were presenting with chronic ADs that were status-post graft repair and the other had an acute type B dissection. In the presented case, neither the neurologist nor the interventional neurologist believed the patient to be an optimal candidate for acute endovascular therapy for his LVO stroke, given his concurrent Stanford type A AD. As the ultimate decision for interventional therapy does not rest with the EP, our case highlights the need to coordinate with the appropriate specialists in order to determine if the patient is a potential candidate for endovascular therapy.

Optimizing $\mathrm{BP}$ in $\mathrm{AD}$ with acute strokes presents another management challenge. The most recent American Heart Association (AHA)/American Stroke Association (ASA) guidelines for the management of acute ADs recommend initial targets of "heart rate less than $60 \mathrm{bpm}$ and a systolic blood pressure between 100 and 120 mmHg" [1]. However, the most recent AHA/ASA guidelines for BP management in acute ischemic stroke recommend avoiding hypotension and maintaining BPs less than $185 / 110 \mathrm{mmHg}$ in patients who are candidates for either tissue plasminogen activator (tPA) or mechanical thrombectomy [17]. The specific BP goals for patients with both ischemic stroke and AD have not been well defined, and should be guided in the ED by discussion with both neurology and cardiothoracic surgery specialists.

The final question raised by these simultaneous pathologies is the order of corrective procedures. In patients with ascending $\mathrm{ADs}$, as was depicted in our case, surgical repair of the $\mathrm{AD}$ is recommended, most commonly through thoracic endovascular repair [1]. However, there is limited literature evaluating ideal operative management in patients with simultaneous $\mathrm{AD}$ and ischemic stroke. A case report in 2017 described a 60year-old male with an ischemic stroke secondary to acute $\mathrm{AD}$, who successfully underwent operative aortic repair five days after presentation and survived with mild residual deficits [18]. Given the overwhelming lack 
of data on therapy for patients with AD leading to LVO stroke, it is vital to involve all pertinent consultants as soon as an $\mathrm{AD}$ leading to ischemic stroke is diagnosed. If a patient has LVO stroke secondary to AD, the interventional neurologist should also be consulted for potential endovascular therapy. Patients with concomitant LVO stroke and $\mathrm{AD}$ are often less than ideal surgical candidates given their coexisting pathologies and the comorbidities contributing to the development of their AD. In the presented case, neither the cardiothoracic surgeon, the neurologist, nor the interventional neurologist thought the patient was a candidate for immediate operative repair or endovascular therapy, and the patient was stabilized and transferred to a medical center with an aortic specialist for potential aortic repair.

\section{Conclusions}

Acute $\mathrm{AD}$ carries a high mortality rate and is often challenging to diagnose. The EP must consider acute $\mathrm{AD}$ in patients presenting with neurologic symptoms secondary to ischemic stroke, especially if they are unable to give a history due to altered mental status or aphasia, as administration of thrombolytics in these patients may be deadly. This case highlights several areas of difficulty in both diagnosing and managing patients with $\mathrm{AD}$ leading to $\mathrm{LVO}$ ischemic stroke. Optimal BP control is unknown, as is the ideal timing of aortic repair and potential interventional therapy for an LVO stroke. As there are limited data on management in this patient cohort, the EP must rapidly engage the neurology, interventional neurology, and cardiothoracic surgery specialists to determine appropriate interventions and timing of operative repair.

\section{Additional Information}

\section{Disclosures}

Human subjects: Consent was obtained by all participants in this study. Conflicts of interest: In compliance with the ICMJE uniform disclosure form, all authors declare the following: Payment/services info: All authors have declared that no financial support was received from any organization for the submitted work. Financial relationships: All authors have declared that they have no financial relationships at present or within the previous three years with any organizations that might have an interest in the submitted work. Other relationships: All authors have declared that there are no other relationships or activities that could appear to have influenced the submitted work.

\section{Acknowledgements}

The view(s) expressed herein are those of the author(s) and do not reflect the official policy or position of Greater San Antonio Emergency Physicians, Brooke Army Medical Center, the U.S. Army Medical Department, the U.S. Army Office of the Surgeon General, the Department of the Army, the Department of the Air Force and Department of Defense or the U.S. Government.

\section{References}

1. Hiratzka LF, Bakris GL, Beckman JA, et al.: 2010 ACCF/AHA/AATS/ACR/ASA/SCA/SCAI/SIR/STS/SVM guidelines for the diagnosis and management of patients with thoracic aortic disease: a report of the American College of Cardiology Foundation/American Heart Association Task Force on Practice Guidelines, American Association for Thoracic Surgery, American College of Radiology, American Stroke Association, Society of Cardiovascular Anesthesiologists, Society for Cardiovascular Angiography and Interventions, Society of Interventional Radiology, Society of Thoracic Surgeons, and Society for Vascular Medicine. Circulation. 2010, 121:266-369. 10.1161/CIR.0b013e3181d4739e

2. Anagnostopoulos CE, Prabhakar MJS, Kittle CF: Aortic dissection and dissecting aneurysms. Am J Cardiol. 1972, 30:263-273. 10.1016/0002-9149(72)90070-7

3. Masuda Y, Yamada Z, Morooka N, Watanabe S, Inagaki Y: Prognosis of patients with medically treated aortic dissections. Circulation. 1991, 84:III7-III13.

4. Hirst AE, Johns VJ, Kime SW: Dissecting aneurysm of the aorta: a review of 505 cases . Medicine. 1958, 37:217-279. 10.1097/00005792-195809000-00003

5. Crawford ES, Kirklin JW, Naftel DC, Svensson LG, Coselli JS, Safi HJ: Surgery for acute dissection of ascending aorta: should the arch be included?. J Thorac Cardiovasc Surg. 1992, 104:46-59. 10.1016/s00225223(19)34835-4

6. Klompas M: Does this patient have an acute thoracic aortic dissection? . JAMA. 2002, 287:2262-2272. 10.1001/jama.287.17.2262

7. Park SW, Hutchison S, Mehta RH, et al.: Association of painless acute aortic dissection with increased mortality. Mayo Clin Proc. 2004, 79:1252-1257. 10.4065/79.10.1252

8. Gaul C, Dietrich W, Friedrich I, Sirch J, Erbguth FJ: Neurological symptoms in type A aortic dissection . Stroke. 2007, 38:292-297. 10.1161/01.STR.0000254594.33408.b1

9. Kawarabuki K, Sakakibara T, Hirai M, et al.: Acute aortic dissection presenting as a neurologic disorder . J Stroke Cerebrovasc Dis. 2006, 15:26-29. 10.1016/j.jstrokecerebrovasdis.2005.09.001

10. Ohle R, Kareemi HK, Wells G, Perry JJ: Clinical examination for acute aortic dissection: a systematic review and meta-analysis. Acad Emerg Med. 2018, 25:397-412. 10.1111/acem.13360

11. Brown MD, Burton JH, Nazarian DJ, Promes SB: Clinical policy: use of intravenous tissue plasminogen activator for the management of acute ischemic stroke in the emergency department. Ann Emerg Med. 2015, 66:322-333.e31.10.1016/j.annemergmed.2015.06.031

12. Kamp TJ, Goldschmidt-Clermont PJ, Brinker JA, Resar JR: Myocardial infarction, aortic dissection, and thrombolytic therapy. Am Heart J. 1994, 128:1234-1237. 10.1016/0002-8703(94)90756-0 


\section{Cureus}

13. Rezaie SR, Swaminathan A, Koyfman A, Long B: A new paradigm shift in acute ischemic stroke, large vessel occlusions, and endovascular therapy. J Emerg Med. 2019, 56:258-266. 10.1016/j.jemermed.2018.10.022

14. Berkhemer OA, Fransen PSS, Beumer D, et al.: A randomized trial of intraarterial treatment for acute ischemic stroke. N Engl J Med. 2015, 372:11-20. 10.1056/NEJMoa1411587

15. Nogueira RG, Jadhav AP, Haussen DC, et al.: Thrombectomy 6 to 24 hours after stroke with a mismatch between deficit and infarct. N Engl J Med. 2018, 378:11-21. 10.1056/NEJMoa1706442

16. Reznik ME, Espinosa-Morales AD, Jumaa MA, Zaidi S, Ducruet AF, Jadhav AP: Endovascular thrombectomy in the setting of aortic dissection. J Neurointerv Surg. 2017, 9:17-20. 10.1136/neurintsurg-2016-012397

17. Powers WJ, Rabinstein AA, Ackerson T, et al.: Guidelines for the early management of patients with acute ischemic stroke: 2019 updates to the 2018 guidelines for the early management of acute ischemic stroke: a guideline for healthcare professionals from the American Heart Association/American Stroke Association. Stroke. 2019, 50:344-418. 10.1161/STR.0000000000000211

18. Morihara R, Yamashita T, Deguchi K, et al.: Successful delayed aortic surgery for a patient with ischemic stroke secondary to aortic dissection. Intern Med. 2017, 56:2343-2346. 10.2169/internalmedicine.8438-16 